\title{
Fluoride-promoted Oxidation of Fischer Alkoxy Carbene Complexes: Stoichiometric and Catalytic Conditions
}

\author{
José Barluenga, ${ }^{\star}$ Facundo Andina, Manuel A. Fernández- \\ Rodríguez, Patricia García-García, Isabel Merino and Enrique \\ Aguilar
}

Instituto Universitario de Química Organometálica "Enrique Moles", Unidad Asociada al C.S.I.C., Universidad de Oviedo, C/ Julián Clavería, 8, 33006 Oviedo, Spain

barluenga@.uniovi.es

\section{SUPPORTING INFORMATION}

Table of Contents for the Suporting Information

General Considerations.

S2

Pentacarbonyl[1-( $\left.1 R^{*}, 3 R^{*}, 4 S^{*}\right)$-menthyloxy-trans-3-

phenyl-2-propenylidene]tungsten(0) $\mathbf{1 e}$.

Fluoride-promoted oxidation reactions of alkoxy carbene complexes 1-3. General Procedure.

$\mathrm{S} 2$

Catalytic fluoride-promoted oxidation reactions of alkoxy carbene complexes 1-3. General Procedure.

$\left(1 R^{*}, 3 R^{*}, 4 S^{*}\right)$-8-Phenylmenthyl trans-3-(2-furyl)prop-2enoate $4 \mathbf{c}$.

$\left(1 R^{*}, 3 R^{*}, 4 S^{*}\right)$-8-Phenylmenthyl trans-3-ferrocenylprop-2enoate $\mathbf{4 d}$

S3

$\left(1 R^{*}, 3 R^{*}, 4 S^{*}\right)$-Menthyl 3-Furylcarboxylate 5c.

${ }^{1} \mathrm{H}-\mathrm{NMR}\left(\mathrm{CDCl}_{3}, 300 \mathrm{MHz}\right)$ spectrum for $\mathbf{5 c}$. S4

${ }^{13} \mathrm{C}-\mathrm{NMR}\left(\mathrm{CDCl}_{3}, 75 \mathrm{MHz}\right)$ spectrum for $\mathbf{5 c}$. S5

${ }^{1} \mathrm{H}-\mathrm{NMR}\left(\mathrm{CDCl}_{3}, 300 \mathrm{MHz}\right)$ spectrum for $4 \mathbf{d} \ldots \ldots . .$. ... S6

${ }^{12} \mathrm{C}-\mathrm{NMR}\left(\mathrm{CDCl}_{3}, 75 \mathrm{MHz}\right)$ spectrum for $\mathbf{4 d}$. 
General Considerations: All reactions were carried out in open-air glass vessels. All common reagents and solvents were obtained from commercial suppliers and used without any further purification unless otherwise indicated. Bu $4 \mathrm{NF}$ was provided by Aldrich as $1 \mathrm{M}$ solution in THF, with a maximum water content of $5 \%$ and was employed with no further purification. Fischer carbene complexes 1a, ${ }^{1} \mathbf{1 b}, \mathbf{c},{ }^{2} \mathbf{1 d},{ }^{3} \mathbf{2 a},{ }^{4} \mathbf{2 b},{ }^{5} \mathbf{2 c},{ }^{6} \mathbf{3 a},{ }^{7} \mathbf{3 b},{ }^{8} \mathbf{3 c}, \mathbf{d},{ }^{9}$ have been previously described and were prepared following established procedures. Hexane, ethyl acetate and diethylether employed in column chromatography were distilled before use. TLC was performed on aluminum-backed plates coated with silica gel 60 with $\mathrm{F}_{254}$ indicator; the chromatograms were visualized under ultraviolet light and/or by staining with a Ce/Mo reagent, or phosphomolibdic acid solutions and subsequent heating. $\mathrm{R}_{f}$ values are reported on silica gel. Flash column chromatography was carried out on silica gel 60, 230-240 mesh. NMR measurements were recorded on Bruker AC200, AC-300 or DPX-300 spectrometers. ${ }^{1} \mathrm{H}$ NMR: splitting pattern abbreviations are: $s$, singlet; bs, broad singlet; d, doublet; t, triplet; q, quartet; m, multiplet; dd, double doublet; $\mathrm{dt}$, double triplet. ${ }^{13} \mathrm{C}$ NMR: multiplicities were determined by DEPT, abbreviations are: $\mathrm{q}, \mathrm{CH}_{3}$; $\mathrm{t}$, $\mathrm{CH}_{2} ; \mathrm{d}, \mathrm{CH} ; \mathrm{s}$, quaternary carbons. Standard pulse sequences were employed for the DEPT experiments. FTIR were performed with a Mattson 3000 FT-IR spectrometer. Mass spectra were determined by Universidad de Oviedo and Universidad de Vigo (CACTI) with a Finnigan Mat95 and a VG AutoSpec M Mass Spectrometers respectively for high resolution mass spectra (HRMS); low resolution mass spectra were obtained with a Hewlett-Packard 5880 A Spectrometer. Electron impact $(70 \mathrm{eV})$ or fast atom bombardment $(\mathrm{FAB})$ techniques were employed. Elemental analyses were carried out with a Perkin-Elmer 240 B microanalyzer.

Spectroscopic and/or physical data for oxidation products $4 \mathbf{a},{ }^{10} \mathbf{4 b},{ }^{11} \mathbf{5 a},{ }^{12} \mathbf{5 b},{ }^{10 \mathrm{~b}} \mathbf{5 d},{ }^{13} \mathbf{6 a},{ }^{14} \mathbf{6} \mathbf{b}^{15}$ and $\mathbf{6 c}, \mathbf{d}^{8}$ have been previously reported.

1 a) Cosset, C.; Del Río, I.; Le Bozec H. Organometallics 1995, 14, 1938-1944. b) Dötz, K. H.; Kuhn, W.; Ackermann, K. Z. Naturforsch., B: Anorg. Chem., Org. Chem. 1983, 38B, 1351-1356.

${ }^{2}$ Barluenga, J.; Montserrat, J. M.; Flórez, J.; García-Granda, S.; Martín, E. Chem. Eur. J. 1995, 1, 236-242.

${ }^{3}$ Barluenga, J.; Fernández-Rodríguez, M. A.; Aguilar, E.; FernándezMarí, F.; Salinas, A.; Olano, B. Chem. Eur. J. 2001, 7, 3533-3544.

${ }^{4}$ Montserrat, J. M. Ph.D. Dissertation, Universidad de Oviedo, 1995.

5 a) Fischer, E. O.; Kreiter, C. G.; Kollmeier, H. J.; Müller, J.; Fischer, R. D. J. Organomet. Chem. 1971, 28, 237-258. b) Fischer, E. O.; Dötz, K. H. J. Organomet. Chem. 1972, 36, C4-C6.

${ }^{6}$ Barluenga, J.; Nandy, S. K.; Laxmi, Y. R. S.; Suárez, J. R.; Merino, I.; Flórez, J.; García-Granda, S.; Montejo-Bernardo, J. Chem. Eur. J. 2003, 9 , 5725-5736.

(a) Chan, K. S.; Wulff, W. D. J. Am. Chem. Soc. 1986, 108, 52295236. (b) Dötz, K. H.; Kuhn, W. J. Organomet. Chem. 1985, 286, C23C26.

${ }^{8}$ Palomero, M. A. Ph.D. Dissertation, Universidad de Oviedo, 2002.

${ }_{9}$ Barluenga, J.; Fernández-Rodríguez, M. A.; Aguilar, E. Org. Lett. 2002, 4, 3659-3662.

${ }^{10}$ a) Balasubramaniyan, V.; Bhatia, G.; Wagh S. B. Tetrahedron 1983, 39, 1475-1485. b) Anders E. Synthesis 1978, 586-588.

${ }^{1}$ Meth-Cohn, O. Org. Syn. 1990, 68, 155-61.

${ }_{12}$ Aoyagi, S.; Tanaka, R.; Naruse, M.; Kibayashi, C. J. Org. Chem. 1998, 63, 8397-8406.
Fischer carbene complex 1e was prepared following established procedures: $:^{2,16}$<smiles>CC(C)[C@H]1CC[C@@H](C)C[C@H]1OC(=[W])C=Cc1ccccc1</smiles>

$1 e$

Pentacarbonyl[1-(1 $\left.R^{*}, 3 R^{*}, 4 S^{*}\right)$-menthyloxy-trans-3phenyl-2-propenylidene]tungsten $(0)$ 1e. $61 \%$ yield. Brown oil. $\mathrm{R}_{f}=0.65$ (Hexane/AcOEt : $\left.5 / 1\right) .{ }^{1} \mathrm{H}$ NMR $\left(\mathrm{CDCl}_{3}, 300 \mathrm{MHz}\right) \delta 8.01(1 \mathrm{H}, \mathrm{d}, J=15.2 \mathrm{~Hz}), 7.67-7.64$ $(2 \mathrm{H}, \mathrm{m}), 7.46-7.44(3 \mathrm{H}, \mathrm{m}), 7.12(1 \mathrm{H}, \mathrm{d}, J=15.2 \mathrm{~Hz})$, $5.04(1 \mathrm{H}, \mathrm{td}, J=3.7$ and $10.4 \mathrm{~Hz}), 2.25-2.21(1 \mathrm{H}, \mathrm{m})$, 2.03-1.84 (4 H, m), 1.75-1.73 (1 H, m), 1.43-1.24 (2 H, m), 1.15-1.02 (7 H, m), $0.91(3 \mathrm{H}, \mathrm{d}, J=6.8 \mathrm{~Hz}) .{ }^{13} \mathrm{C} \mathrm{NMR}$ $\left(\mathrm{CDCl}_{3}, 75 \mathrm{MHz}\right) \delta 302.0(\mathrm{~s}, \mathrm{C}=\mathrm{W}), 203.3$ (s, CO), 197.6 (s, 4 CO), 143.0 (d), 134.5 (d), 132.0 (d), 130.7 (s), 129.12 (d, $2 \mathrm{CH}), 129.07$ (d, $2 \mathrm{CH}), 93.7$ (d), 48.1 (d), 42.0 (t), $34.1(\mathrm{t}), 31.1(\mathrm{~d}), 26.8(\mathrm{~d}), 24.1(\mathrm{t}), 21.9(\mathrm{q}), 21.4(\mathrm{q}), 17.3$ (q). MS (m/z) 454 (M-5 CO, 4), 370 (18), 138 (24), 95 (100); HRMS (EI) calcd for $\mathrm{C}_{24} \mathrm{H}_{26} \mathrm{O}_{6} \mathrm{~W}, 594.1233$. Found: 594.1263.

Fluoride-promoted oxidation reactions of alkoxy carbene complexes 1-3. General Procedure: 1.5 equivalents of the fluoride source (see Table 2) were added at room temperature to a solution of the corresponding Fischer carbene complex 1-3 (1 mmol) in 1,2-DME (30 $\mathrm{mL}$ ). The reaction was stirred at that temperature and monitored by TLC. When disappearance of the starting material was observed, silica gel $(1 \mathrm{~g})$ was added, solvent was evaporated under vacuum and the residue was purified by flash chromatography to obtain the corresponding ester in the yield listed in the Tables.

Catalytic fluoride-promoted oxidation reactions of alkoxy carbene complexes 1-3. General Procedure: 2-10 mol \% of $\mathrm{Bu} 4 \mathrm{NF}$ (see Table 3) were added at room temperature to a solution of the corresponding Fischer carbene complex 1-3 (1 mmol) in 1,2-DME (30 mL). The reaction was stirred at that temperature and monitored by TLC. When disappearance of the starting material was observed, silica gel (1 g) was added, solvent was

${ }^{13}$ Spasov, S.; Simova, S. J. Chem. Soc. Perkin Trans 2 1978, 1113 1114 .

a) Foot, J. S.; Kanno, H.; Giblin, G. M. P.; Taylor, R. J. K. Synthesis, 2003, 1055-1064. b) Nilsson, K.; Ullenius, C.; Krause, N. J. Am. Chem Soc. 1996, 118, 4194-4195.

15 Buchert, M.; Hoffmann, M.; Reissig, H.-U. Chem Ber. 1995, 128, 605-614.

16 a) Södeberg, B. C.; Hegedus, L. S.; Sierra, M. A. J. Am. Chem. Soc. 1990, 112, 4364-4374. b) Semmelhack, M. F.; Bozell, J. J. Tetrahedron Lett. 1982, 23, 2931-2934. c) Fischer, E. O.; Selmayr, T.; Kreissl, F. R. Chem. Ber. 1977, 110, 2947-2955. d) Fischer, E. O.; Selmayr, T.; Kreissl, F. R. Monast. Chem. 1977, 108, 759-765; Chem. Abstr. 1977, 87, 201659a. 
evaporated under vacuum and the residue was purified by flash chromatography to obtain the corresponding ester in the yields listed in Table 3 .<smiles>CC1CCC(C(C)(C)c2ccccc2)C(OC(=O)/C=C/c2ccco2)C1</smiles>

$\left(1 R^{*}, 3 R^{*}, 4 S^{*}\right)-8$-Phenylmenthyl

trans-3-(2furyl)prop-2-enoate 4c. $61 \%$ yield (with $\mathrm{KF}$ ); $65 \%$ yield (with Bu4NF). Yellow oil. $\mathrm{R}_{f}=0.53$ (Hexane/Et $t_{2} \mathrm{O}: 5 / 1$ ). ${ }^{1} \mathrm{H} \mathrm{NMR}\left(\mathrm{CDCl}_{3}, 300 \mathrm{MHz}\right) \delta 7.45(1 \mathrm{H}, \mathrm{d}, J=2.0 \mathrm{~Hz})$, 7.41-7.06 (5 H, m), $6.84(1 \mathrm{H}, \mathrm{d}, J=15.7 \mathrm{~Hz}), 6.51(1 \mathrm{H}$, $\mathrm{d}, J=3.4 \mathrm{~Hz}), 6.44(1 \mathrm{H}, \mathrm{dd}, J=2.0$ and $3.4 \mathrm{~Hz}), 5.80(1$ $\mathrm{H}, \mathrm{d}, J=15.7 \mathrm{~Hz}), 4.87(1 \mathrm{H}, \mathrm{dt}, J=4.3$ and $10.5 \mathrm{~Hz})$, 2.14-0.87 $(17 \mathrm{H}, \mathrm{m}) .{ }^{13} \mathrm{C} \mathrm{NMR}\left(\mathrm{CDCl}_{3}, 75 \mathrm{MHz}\right) \delta 166.1$ (s, C=O), 151.6 (s), 150.9 (s), 144.4 (d), 130.4 (d), 127.9 (d, 2 CH), 125.3 (d, 2 CH), 124.8 (d), 116.2 (d), 114.3 (d), 112.0 (d), 74.4 (d), 50.5 (d), $41.6(\mathrm{t}), 39.5(\mathrm{~s}), 34.5$ (t), 31.2 (q), 27.9 (d), 26.5 (t), 24.8 (q), 21.7 (q). Elemental analysis calcd (\%) for $\mathrm{C}_{23} \mathrm{H}_{28} \mathrm{O}_{3}$ (352.47): $\mathrm{C} 78.38, \mathrm{H} \mathrm{8.01}$; found $\mathrm{C}$ 78.25, H 7.97 .

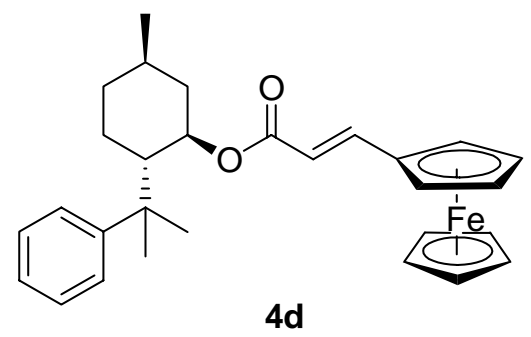

$\left(1 R^{*}, 3 R^{*}, 4 S^{*}\right)-8$-Phenylmenthyl

trans-3ferrocenylprop-2-enoate 4 d. $62 \%$ yield. Red oil. $\mathrm{R}_{f}=$ 0.55 (Hexane/Et $2 \mathrm{O}: 5 / 1) .{ }^{1} \mathrm{H}$ NMR $\left(\mathrm{CDCl}_{3}, 300 \mathrm{MHz}\right) \delta$ 7.31-7.24 (3 H, m), 7.21 (1 H, d, J=15.8 Hz), 7.20-7.15 (2 $\mathrm{H}, \mathrm{m}), 5.43(1 \mathrm{H}, \mathrm{d}, J=15.8 \mathrm{~Hz}), 4.87(1 \mathrm{H}, \mathrm{td}, J=4.0$ and $10.5 \mathrm{~Hz}), 4.38$ (4 H, bs), 4.14 (5 H, bs), 2.11-0.87 (17 H, m). ${ }^{13} \mathrm{C} \mathrm{NMR}\left(\mathrm{CDCl}_{3}, 75 \mathrm{MHz}\right) \delta 166.1(\mathrm{~s}, \mathrm{C}=\mathrm{O}), 151.6$ (s), 144.6 (d), 127.8 (d, 2 CH), 125.3 (d, 2 CH), 124.7 (d), 115.6 (d), 78.7 (s, Cp1), 74.0 (d), 70.5 (d, 2 CH, Cp1), 69.4 (d, 5 CH, Cp2), 68.4 (d, CH, Cp1), 68. 3 (d, CH, Cp1), 50.5 (d), 41.6 (t), 39.6 (s), 34.5 (t), 31.2 (d), 27.4 (q), 26.5 (t), 25.3 (q), 21.7 (q). MS (m/z) $470\left(\mathrm{M}^{+}, 20\right), 256$ (46), 149 (92), 119 (100); HRMS (EI) calcd for $\mathrm{C}_{29} \mathrm{H}_{34} \mathrm{O}_{2} \mathrm{Fe}, 470.1908$. Found: 470.1923.<smiles>CC(C)[C@H]1CC[C@@H](C)C[C@H]1OC(=O)c1ccoc1</smiles>

$5 c$

$\left(1 R^{*}, \mathbf{3} R^{*}, \mathbf{4} S^{*}\right)$-Menthyl 3-Furylcarboxylate $\mathbf{5 c} .73 \%$ yield. Orange oil. $\mathrm{R}_{f}=0.46\left(\mathrm{Hexane}_{\mathrm{Et}} \mathrm{O}: 5 / 1\right)$. ${ }^{1} \mathrm{H}$ NMR $\left(\mathrm{CDCl}_{3}, 300 \mathrm{MHz}\right) \delta 8.00(1 \mathrm{H}, \mathrm{bs}), 7.41$ (1 H, bs), 6.74 (1 $\mathrm{H}, \mathrm{bs}), 4.86(1 \mathrm{H}, \mathrm{dt}, J=4.3$ and $10.8 \mathrm{~Hz}), 2.08(1 \mathrm{H}, \mathrm{m})$, $1.92(1 \mathrm{H}, \mathrm{m}), 1.70(2 \mathrm{H}, \mathrm{m}), 1.47(2 \mathrm{H}, \mathrm{m}), 1.12-0.89$ (9 $\mathrm{H}, \mathrm{m}), 0.79(3 \mathrm{H}, \mathrm{d}, J=6.8 \mathrm{~Hz}) \cdot{ }^{13} \mathrm{C} \mathrm{NMR}\left(\mathrm{CDCl}_{3}, 75\right.$ $\mathrm{MHz}) \delta 162.6$ (s, C=O), 147.3 (d), 143.4 (d), 119.8 (s, Ar), 109.8 (d), 74.1 (d), 47.0 (d), $40.8(\mathrm{t}), 34.1(\mathrm{t}), 31.3$ (d), 26.4 (d), 23.6 (t), 21.9 (q), 20.5 (q), 16.5 (q). MS ( $/ \mathrm{m} / \mathrm{z}) 138$ (50), 95 (100); HRMS (EI) calcd for $\mathrm{C}_{15} \mathrm{H}_{22} \mathrm{O}_{3}, 250.1563$. Found: 250.1572. 
$108 L^{\circ} 0$ $6208^{\circ} 0-$ $2068^{\circ} 0$

$6216^{\circ} 0$

$297.6^{\circ} 0-$

$2000^{\circ}$

$00+0^{\circ}$

$68 \angle 9$

$\angle 0 Z 1 " 1-$

$\angle Z E D^{\circ}$

$92 \angle D \cdot 1$

$9605^{\circ}$ !

$1789^{\circ} 1-$

दर68:-

SSI6:- $/=$

GटरE : -

ZSOI'

$68: 8 \div$

ZCE 8 -

$0 \rightarrow 58$ क

$\varepsilon 898^{\circ} t$

$1068^{\prime \prime} t$

s $706^{\circ} \rightarrow$

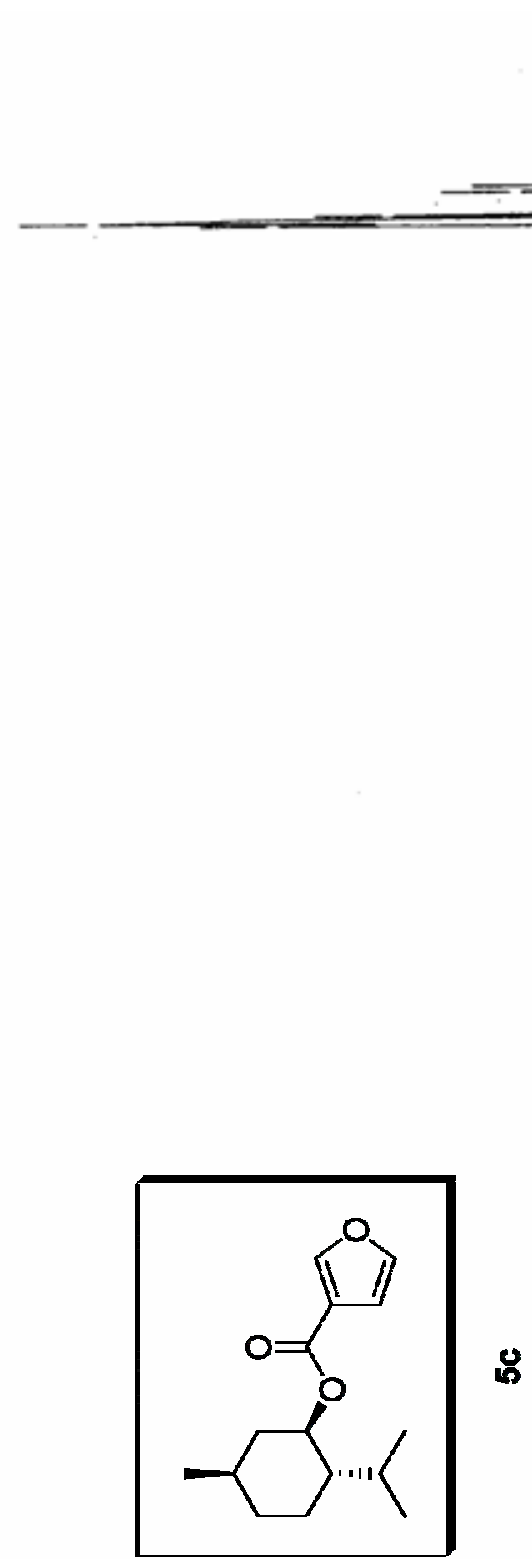

$90+\angle ' 9-$

$2110^{\circ} \mathrm{L}$

8

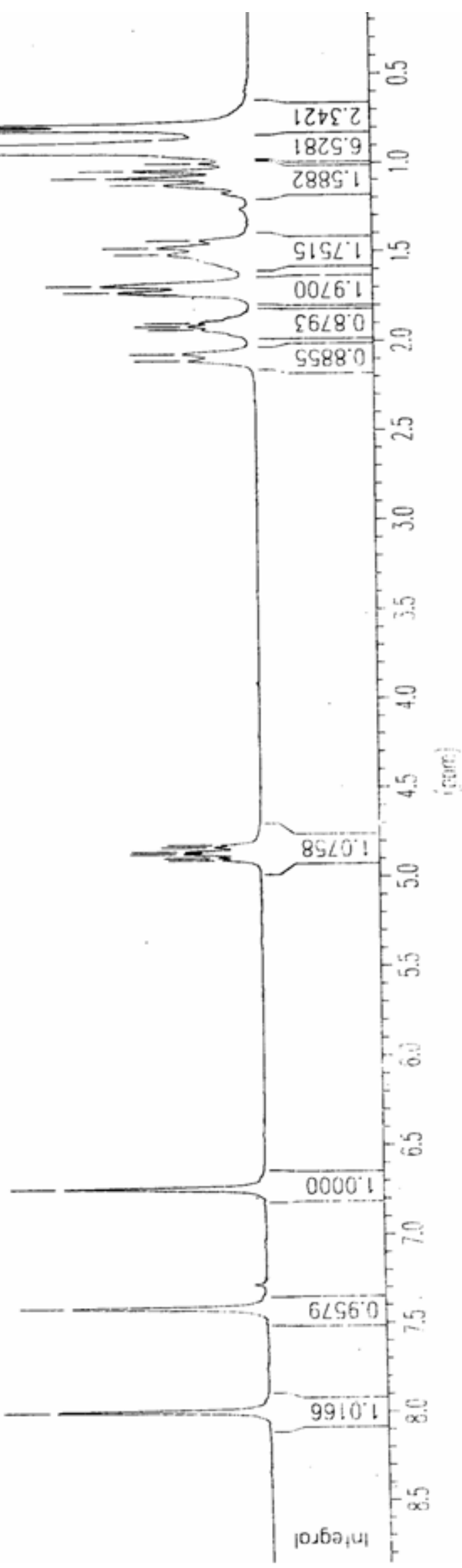




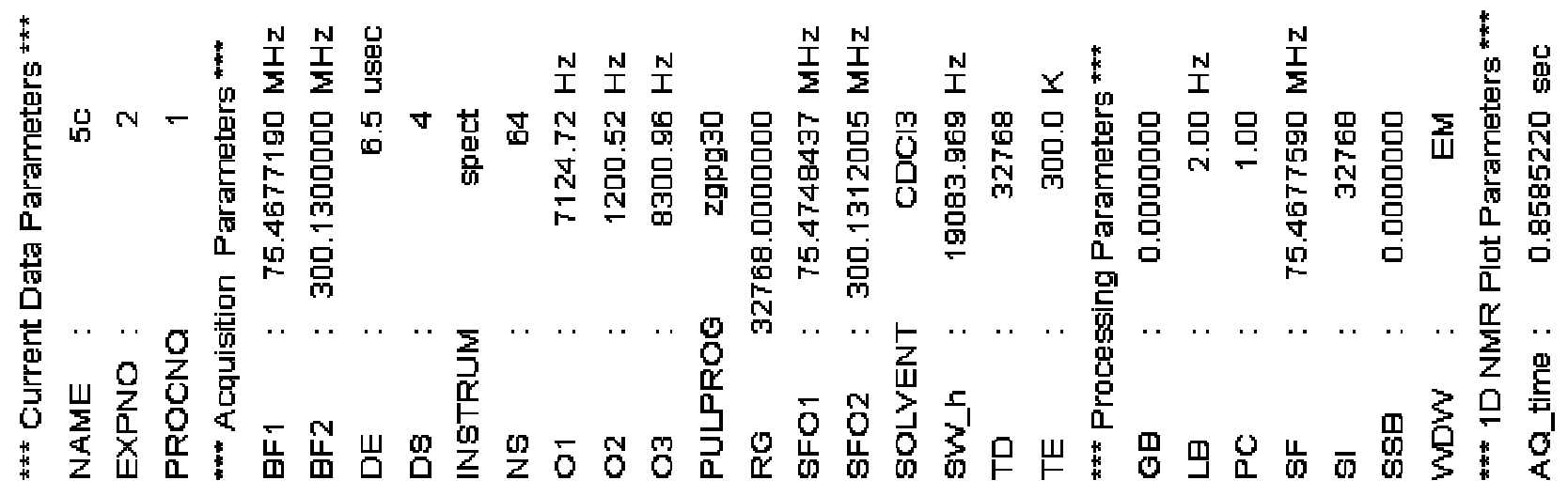
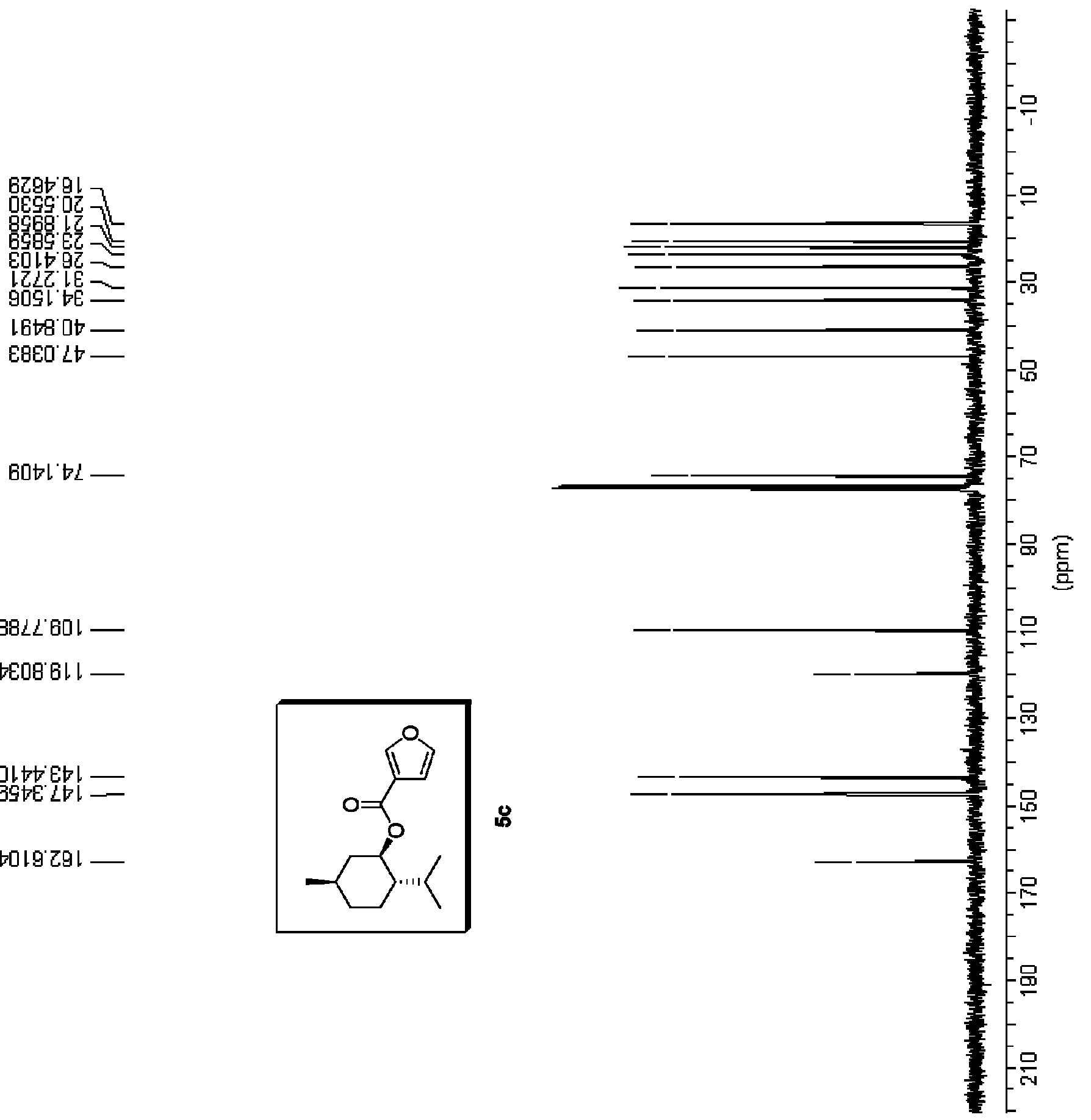

$88 L \angle 60 レ-$

†E086!. -

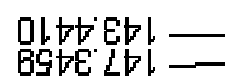

$\operatorname{tolg} 291$

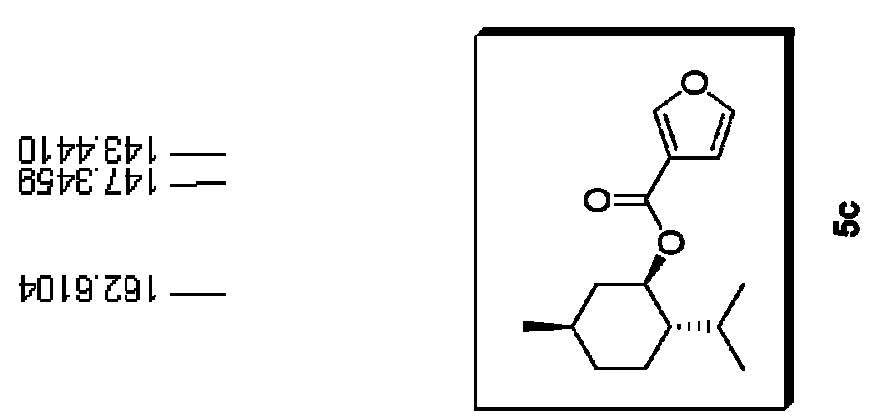

$60 t h b 2$ 


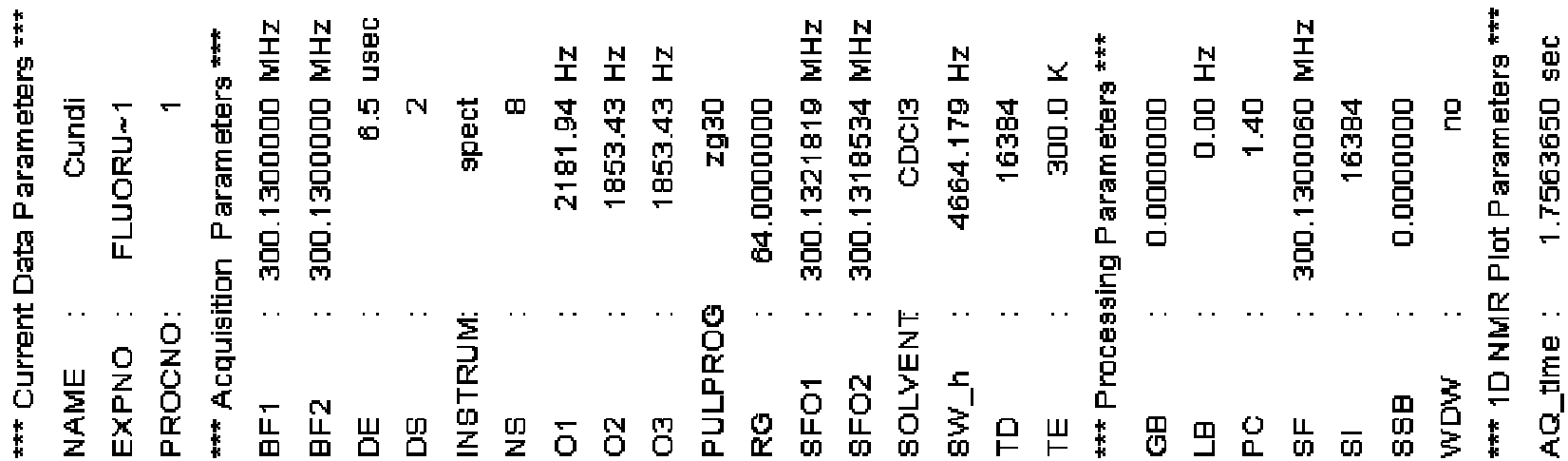

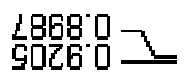
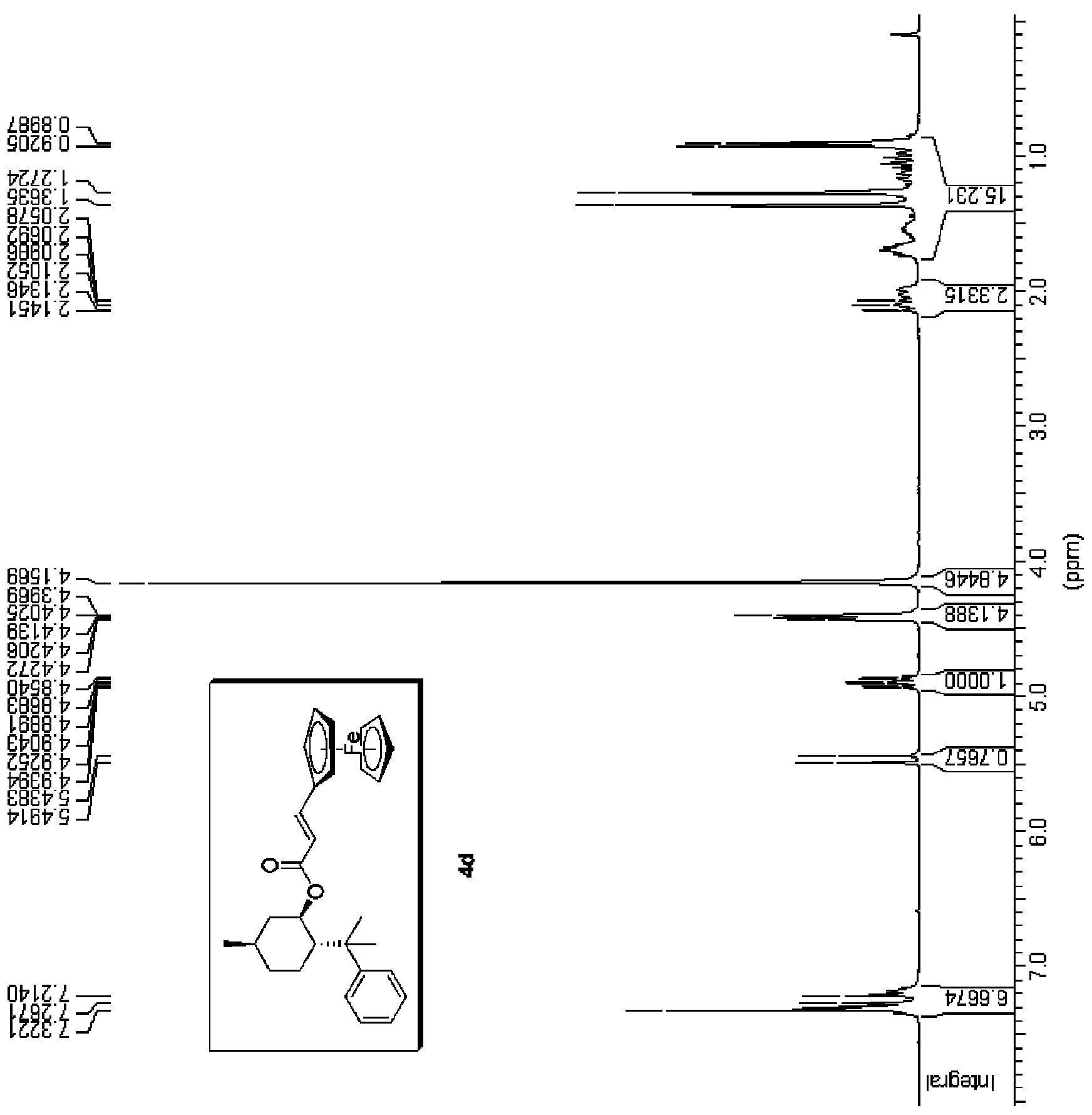


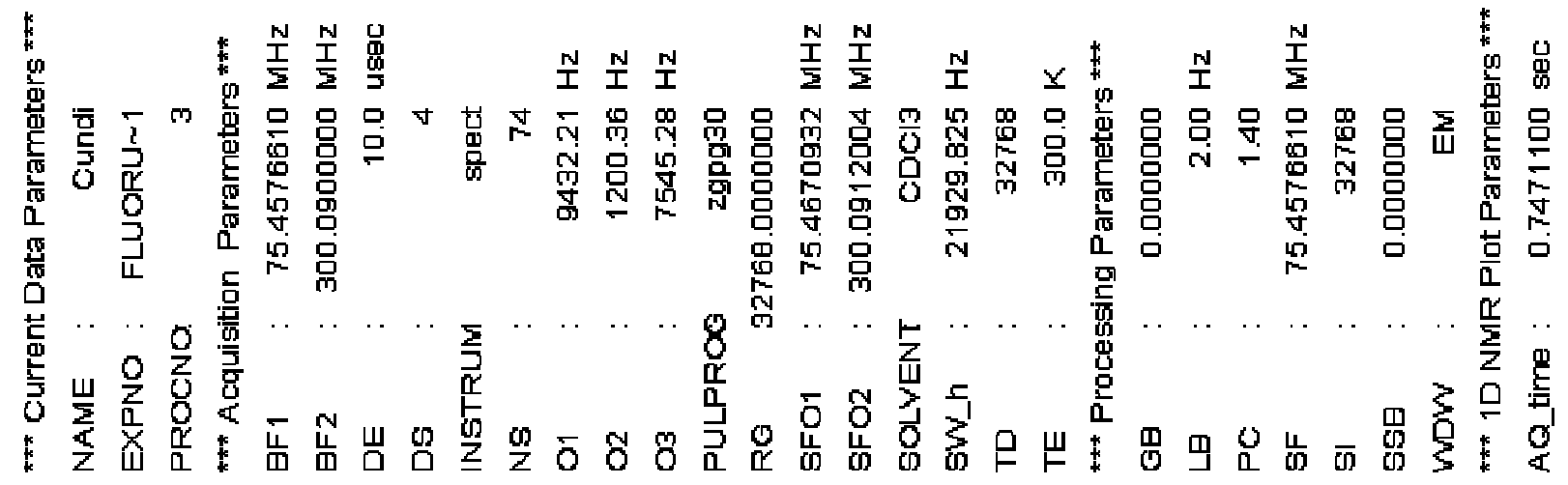

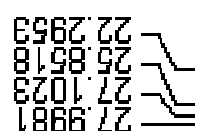

gosi LE

$211 \cdot 98=$

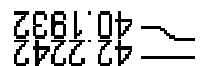

LLEL'IS-

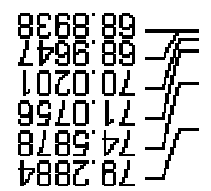

LBEL'GI. -

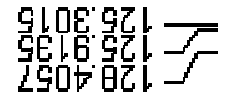

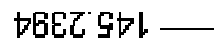

LEZZGI-
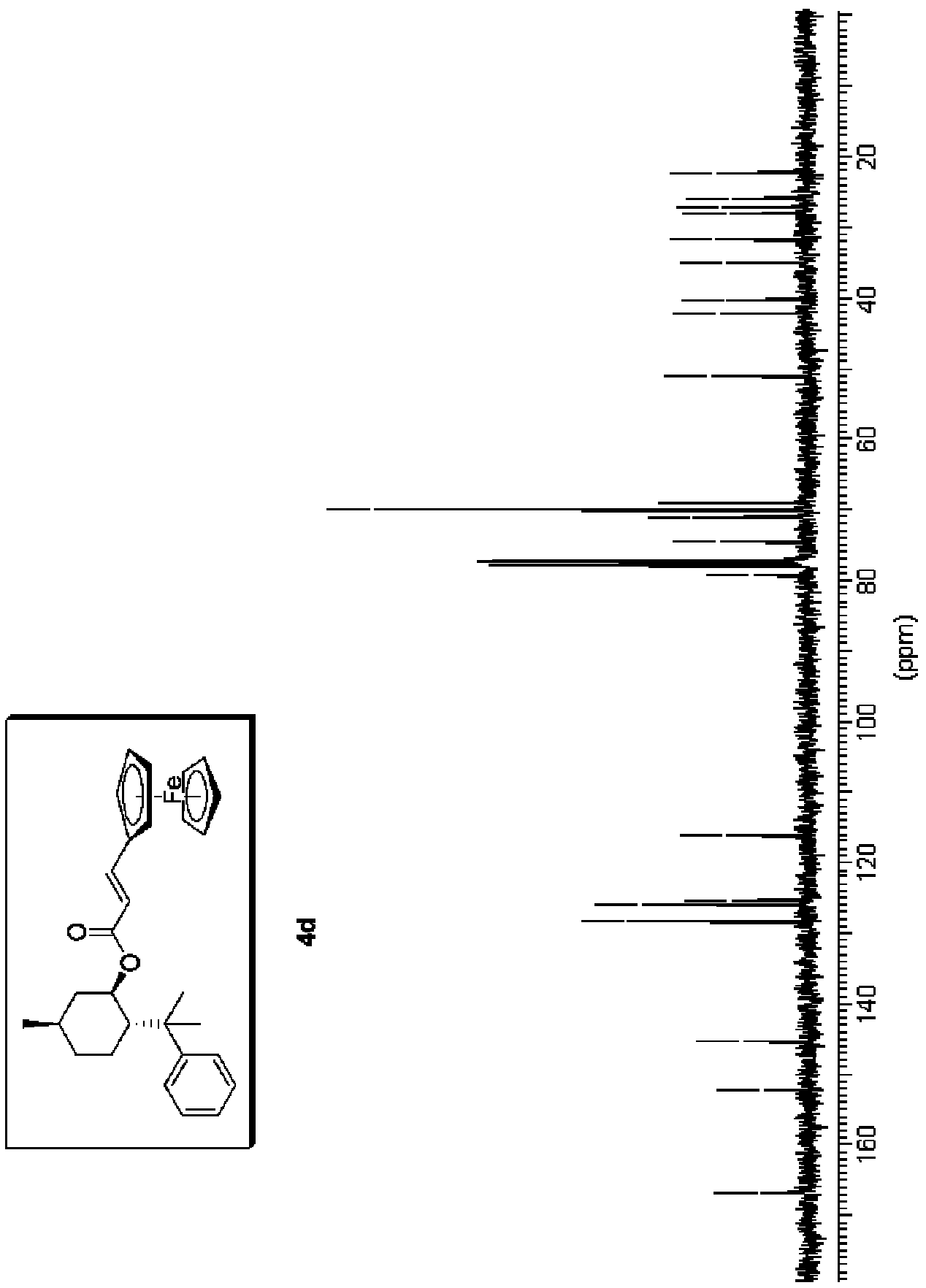

699L99l- 